\title{
A Multi-spectral Image Fusion Algorithm Based on PCA and Red-Black Wavelet
}

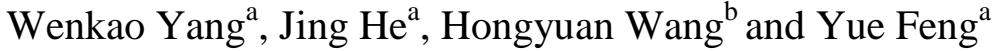 \\ ${ }^{a}$ Beijing Jiaotong University, Department of Electronic and Information \\ Engineering, Beijing, China, 100044,wkyang@bjtu.edu.cn \\ 13120070@bjtu.edu.cn13120064@bjtu.edu.cn \\ ${ }^{b}$ Dalian Polytechnic University, School of information science and engineering, \\ Dalian, China,116034, yuan1096018567@126.com
}

\begin{abstract}
A multi-spectral image fusion algorithm based on red-black wavelet (RBW) and principal component analysis (PCA) is proposed in this paper to enhance the image performance. The PCA algorithm was used to extract the diverse features and details of the multi-spectral image, and, then, these features were decomposed by RBW and fused by the improved diverse algorithms using low-frequency and high-frequency coefficients at different scales, frequency domains, decomposition layers, and frequency bands. Finally, these fused features and multi-spectral images were reconstructed by $R B W$ and PCA inversion. The results of our experiments showed that the proposed algorithm provided higher spatial resolution and more excellent spectral information. In addition, it improved the processing speed, took less memory, and offers the potential for real-time processing.
\end{abstract}

Keywords: Multi-spectral image fusion, Red-black wavelet transform, PCA algorithm, Fusion strategy

\section{Introduction}

Although a multi-spectral image has excellent spectral information, its spatial resolution is usually poor. It is a crucially important to enhance remote sensing images by fusing multi-spectral images with a high-resolution satellite pour l'observation de la terre (SPOT) panchromatic image to get an image with both excellent spectral information and high spatial resolution. Image fusion is a combined process of a plurality of image information of the same scene, which is captured by diverse image sensors working by different mechanisms[1]. The process is conducted on three levels, i.e., pixel fusion, feature fusion and decision fusion, according to the degree to which information is to be extracted. Pixel-level fusion is the most basic and extensively used application area in remote sensing image fusion[2], and the representative methods are principal component analysis (PCA)[3], intensity-hue-saturation method (IHS)[4], discrete wavelet transform (DWT)[5], wavelet transform combined with PCA6, and Brovey[7]. The PCA algorithm increases the space expressiveness of the fusion image and provides good, detailed performance, but it decreases the spectral resolution. Although the IHS method provides excellent high-frequency information, it results in a serious loss of spectral information. Conversely, DWT provides good spectral information at the cost of relatively low spatial resolution[8]. The brovey transform is a simpler algorithm, but it is used mainly to deal with three-band, multi-spectral data.

To overcome the issues of lower spatial resolution and spectral information induced by the methods discussed above, many algorithms have been developed to improve the performance of the infusion image, such as the combination of wavelet transform and IHS 
transform [9-10], lifting wavelet transform [11], and curvelet transform [12]. The algorithms that are based on separate wavelet transform have been used extensively in image processing due to their low computational complexity. They enhance the information in both the horizontal and vertical directions, but they are not sensitive enough to other directions, such as the diagonal direction, which is a common disadvantage of the separate wavelet transform-based algorithms. The important information related to images generally is concentrated in the area called the "diamond-type" area in the two-dimensional spectrum domain, and two-dimensional, non-separable wavelet algorithms are good at dealing with the information. The lifting wavelet transform based on five plant morphology sampling is a representative, non-separable wavelet proposed by Geert Uytterhoeven [13], and it is also called the red-black wavelet (RBW) transform. It can eliminate the correlation in the row and column directions and decrease the correlation in the diagonal direction; in addition, it has the advantage that the separable algorithm based on lifting scheme provides, such as decomposition algorithm combination with reconstruction algorithm and lower computation memory. However, its ability to maintain spatial detail is still weak.

A novel algorithm based on the RBW transform and the PCA algorithm is proposed in this paper to fuse multi-spectral images. The algorithm has the advantages of both the RBM transform and the PCA algorithm, e.g., high compactness, orthogonality, and good directivity. The PCA algorithm extracts the diverse features and details of the multi-spectral image, and the RBW transform decomposes these salient features for different scales, frequency domains, decomposition layers, and frequency bands. These features are fused by the new diverse algorithms. Then, the fused images at different scales, frequency domains, decomposition layers, and frequency bands are reconstructed to obtain an image with higher spatial and spectral resolution. The strategy that the proposed algorithm based on the RBW transform and the PCA algorithm uses is very similar to human retinas that deal with images at various frequency channels, and it can obtain better visual performance. The results of our experiments with the proposed algorithm indicated that the comprehensive performance of the proposed algorithm outperforms other such algorithms.

The remainder of the paper is organized as follows. Section 2 introduces the image fusion application of the PCA algorithm. The RBW transform is discussed in detail in Section 3. Section 4 describes the new fusion algorithm in detail. The simulation results and parametric analysis are presented in Section 5, and our conclusions are presented in Section 6.

\section{Red-Black Wavelet Transform}

The RBW transform is constructed by two lifting steps, i.e., horizontal/vertical lifting and diagonal lifting. Each ascending wavelet transform is conducted in three steps, i.e., decomposition, prediction, and updating[13]. In the following, we chose an $8 \times 8$ image block $X(i, j)$ to illustrate these steps.

\subsection{Horizontal/Vertical Lifting}

Splitting: Split the image $X(i, j)$ into the red block $\gamma_{i}$ and the black block $\beta_{i}$ according to the horizontal and vertical directions, as shown in Figure. 1(a). 


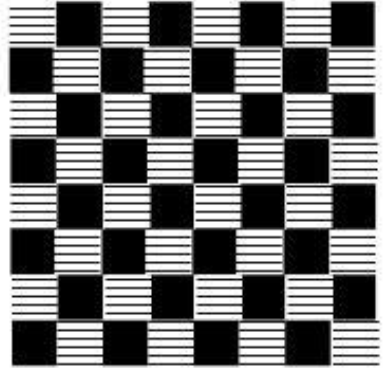

Figure 1. (a) Splitting

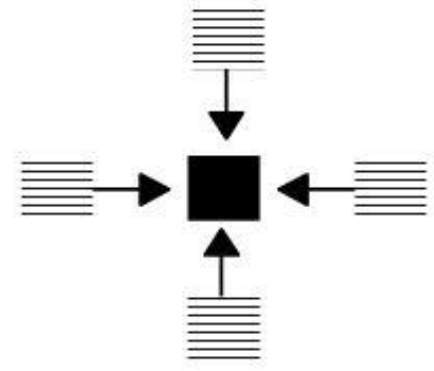

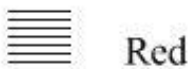

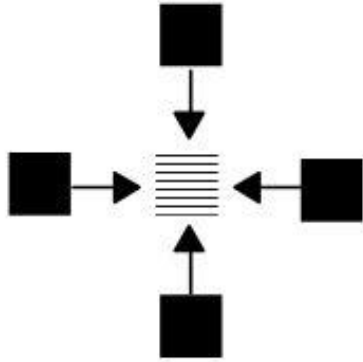

Blue

Prediction: Predict the values of the black squares based on the values of their immediate four neighboring red squares and replace the original value of black squares with the predictive value. As a result, the details of the image are stored in the black block. The prediction is given as:

$$
\begin{aligned}
& X(i, j) \leftarrow X(i, j)-\frac{1}{4}\left(\begin{array}{l}
X(i-1, j)+X(i+1, j)+ \\
X(i, j+1)+X(i, j-1)
\end{array}\right) \\
& (i \bmod 2 \neq j \bmod 2)
\end{aligned}
$$

Update: As shown in Figure. 1(c), the red block is updated with the four neighboring black squares to preserve the mean value. As a result, the approximate information of the image is stored in the red block. The updating is expressed in Formula (2).

$$
\begin{aligned}
& X(i, j) \leftarrow X(i, j)+\frac{1}{8}\left(\begin{array}{l}
X(i-1, j)+X(i+1, j)+ \\
X(i, j+1)+X(i, j-1)
\end{array}\right) \\
& (i \bmod 2=j \bmod 2)
\end{aligned}
$$

\subsection{Diagonal Lifting}

In the diagonal direction, the checkerboard is considered to be rotated by $45^{\circ}$.

Splitting: Split the red squares $\gamma_{i}$ into two parts, i.e., blue squares $\gamma_{i-1}$ and yellow squares $\beta_{i-1}$, as shown in Figure. 2(a).
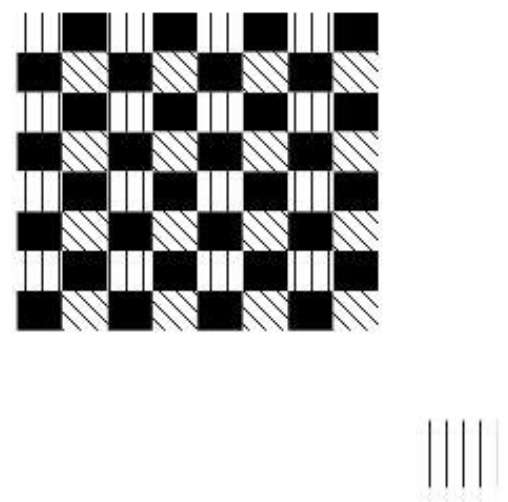

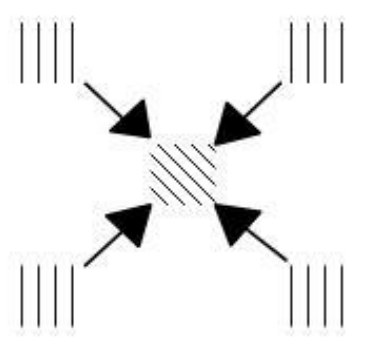

Blue

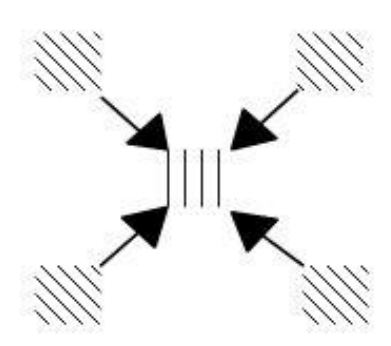

Yellow
Figure 2. (a) Splitting (b) Prediction (c) Updating 
Prediction: Predict the values of the yellow squares based on the values of their immediate four neighboring blue squares, and save the predicted deviation in the yellow block. The prediction is given as:

$$
\begin{aligned}
& X(i, j) \leftarrow X(i, j)-\frac{1}{4}\left(\begin{array}{l}
X(i-1, j-1)+X(i+1, j+1)+ \\
X(i-1, j+1)+X(i+1, j-1)
\end{array}\right) \\
& (i \bmod 2=1 \text { and } j \bmod 2=1)
\end{aligned}
$$

Update: Update the blue squares using the four neighboring (new) yellow squares to preserve the mean value, as shown in Figure. 2(c). The updating is expressed as:

$$
\begin{aligned}
& X(i, j) \leftarrow X(i, j)+\frac{1}{8}\left(\begin{array}{l}
X(i-1, j-1)+X(i+1, j+1)+ \\
X(i-1, j+1)+X(i+1, j-1)
\end{array}\right) \\
& (x \bmod 2=0 \text { and } j \bmod 2=0)
\end{aligned}
$$

Reordering: The above transform is $100 \%$ in place and all coefficients will be interleaved, so we should reorder the coefficients in order to facilitate multi-resolution. One of the powerful features of the lifting scheme is that it does not require additional memory to calculate the forward or inverse transform[14 15].

According to Eq. (4) and Eq. (5), we can obtain the system function diagrams of the RBW transform, which functions as a low-frequency filter in Figure.3(a) and a high-frequency filter in Figure. 3(b).

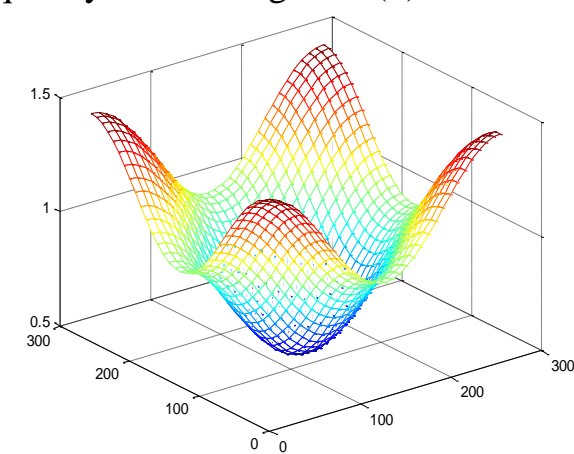

(a)

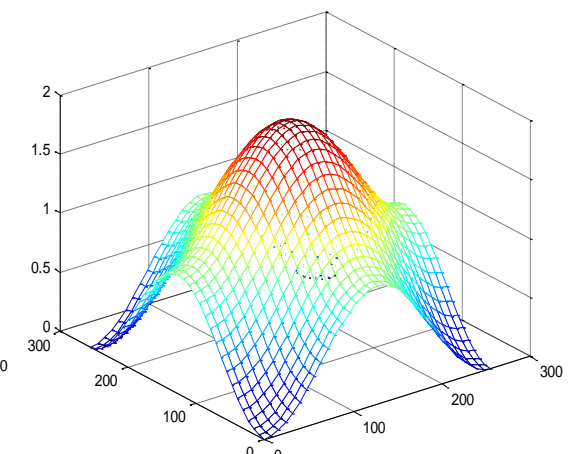

(b)

Figure 3. System Function Diagrams of the RBW Transform: (a) Prediction; (b) Updating

\section{PCA- and RBW-based Multi-spectral Image Fusion}

Multi-spectral image and SPOT panchromatic image fusion is one of the main modes of remote images fusion. The multi-spectral image provides the spectrum in detail, while the panchromatic image has good spatial resolution. By fusing the multi-spectral image and the SPOT panchromatic image, the spectral characteristics are retained and the spatial resolution of the multi-spectral image is improved, providing a fusion image that contains extensive information.

The PCA algorithm, which is available to all bands of the multi-spectral image, can extract the overall characteristics of the images. The RBW transform is a non-separable wavelet based on the five plant morphology sampling wavelet lifting scheme, which has the significant advantages of maintaining high spatial resolution and having extensive information concerning the colors of multi-spectral images. In this paper, we describe the innovative combination of the RBW wavelet and the PCA algorithm for multi-spectral image fusion. The principal components of the two source images are decomposed into high-frequency parts and low-frequency parts, and, then, they are fused with a new weighted method based on a regional feature, local energy, and an improved method 
based on neighborhood spatial frequency. For RBW wavelet, both the forward and inverse transforms are very simple to implement and have fast execution times. Thus, this algorithm can ensure more information in order to achieve the optimal fusion of the details of the image and simultaneously reduce the computation time.

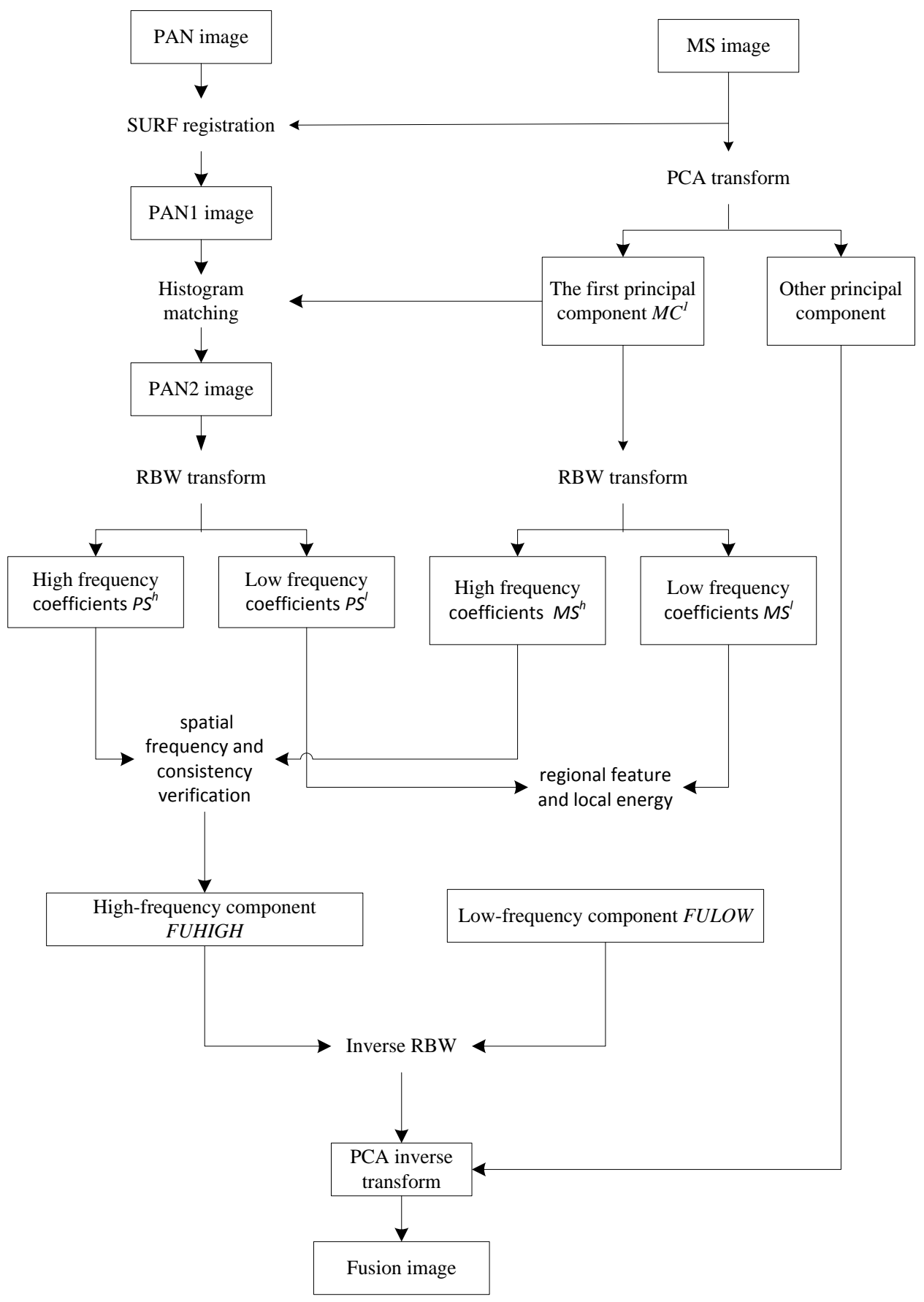

Figure 4. Experimental Procedure

A schematic diagram of the panchromatic (PAN) and multi-spectral (MS) image fusion step is shown above, and the fusion steps are as follows:

(1). Register the high-resolution PAN image with the multi-spectral MS image by using the SURF-based algorithm registration method to get the image PAN1. 
(2). Perform PCA of the MS image to obtain the first component $M C^{1}$ and other components. $M C^{1}$ and the PAN1 image are processed with histogram matching [17] to get the enhanced PAN2 image.

(3). Apply the RBW transform to the PAN2 image and $M C^{1}$ to get the high-frequency coefficients, $P S^{h}$ and $M S^{h}$, and the low-frequency coefficients, $P S^{l}$ and $M S^{l}$;

(4). The coefficients that represent low frequencies commonly are processed by the weighted average method 16 . However, this method measures the fusion coefficients only by their individual coefficients, which will inevitably lead to blurring the edge of the fused image and reducing the accuracy. In order to preserve the fidelity of the image and make better use of coherency between the sub-bands transformed by the lifting wavelet, we propose a weighted method based on the regional feature and local energy to obtain the low-frequency coefficient $F U L O W$ of the fusion image.

Set $\psi(M, b)$ and $\psi(P, b)$ as the wavelet coefficients of $M S^{l}$ and $P S^{l}$, respectively, at the pixel $b(i, j)$. Note that $\bar{\mu}(M, d)$ represents the mean value of $\psi(M, b)$ in the $W$ domain, where $W$ indicates the $3 \times 3$ domain of point $b$. The equation $E_{M}=\sum_{(i, j) \in W} G_{M}(i, j)$ denotes the neighborhood energy of the $W$ domain.

First, the matching matrix is defined as Eq. (5)

$$
M(b)=\frac{2 \sum_{d \in D} \alpha(d)|\psi(M, d)-\psi(M, b)||\psi(P, d)-\psi(P, d)|}{H(M, d)+H(P, b)}
$$

The point in the matching matrix ranges from 0 to 1 . The closer to 1 the variable is, the higher correlation is between the two sets of low-frequency images. Then, a local variance is given as Eq. (6).

$$
H(M, b)=\sum_{b \in D} \alpha(d)|\psi(M, b)-\bar{\mu}(M, d)|^{2}
$$

where $\alpha(d)$ is the weight, which meets the conditions of the formula:

$$
\sum_{d \in D} \alpha(d)=1
$$

The nearer $d$ is to $b$, the larger the weight is. Similarly, we can get $H(P, b)$.

Also, the match threshold $T$ is defined, and it ranges from 0.5 to 1 .

If $M(b) \leq T$,

$\psi(F U L O W, b)=\lambda_{M} \psi(M, b)+\lambda_{P} \psi(P, d)$

else,

$$
\psi(F U L O W, b)=\left\{\begin{array}{l}
\psi(M, b), H(M, b)>H(P, b) \\
\psi(P, b), H(M, b) \leq H(P, b)
\end{array}\right.
$$

Where,

$$
\begin{gathered}
\lambda_{M}=\frac{E_{M}}{E_{M}+E_{P}} \\
\lambda_{M}+\lambda_{P}=1
\end{gathered}
$$

(5). To take advantage of the features of the high-frequency coefficients lifted by the RBW transform, we used an improved fusion rule based on neighborhood spatial 
frequency and consistency verification to obtain the new high-frequency coefficient, FUHIGH . The specific steps are as follows:

(1) In the space $W$, compute the neighborhood spatial frequency of each pixel, which is from sub-images corresponding to decomposed layers of the source image MS, PAN, respectively [17].

(2) Compare neighborhood spatial frequency of the sub-images and composite corresponding sub-images to the fusion image,

$$
G_{F}(i, j, l)=\left\{\begin{array}{l}
G_{P}(i, j, l), S F_{P}(i, j, l)>S F_{M}(i, j, l) \\
G_{M}(i, j, l), S F_{M}(i, j, l)>S F_{P}(i, j, l)
\end{array}\right.
$$

else $S F_{M}(i, j, l)=S F_{P}(i, j, l)$,

$$
G_{F}(i, j, l)=\left\{\begin{array}{l}
G_{P}(i, j, l), G_{P}(i, j, l)>G_{M}(i, j, l) \\
G_{M}(i, j, l), G_{M}(i, j, l)>G_{P}(i, j, l)
\end{array}\right.
$$

where $S F_{M}(i, j, l), S F_{P}(i, j, l)$ represent spatial frequency at point $(i, j)$ that belongs to the $l$ layer of sub-images corresponding to the source image MS, PAN, while $G_{M}(i, j, l), G_{P}(i, j, l)$, and $G_{F}(i, j, l)$ denote the coefficients that correspond to the sub-image of the source image MS, PAN and the fused image FHIGH.

(3) Verify consistency. Slide on the fusion image with a $3 \times 3$ window and verify the center pixel with its surrounding pixels. For example, if the center pixel comes from sub-images of the source image PAN, while its surrounding pixels come from sub-images of the source image MS, then change the center pixel's values with coefficients that correspond to the sub-images of MS.

For an $m \times n$ image, where $m$ and $n$ represent the number of rows and columns, respectively, the overall activity level in the image, which is denoted by SF, may be expressed as,

$$
S F=\sqrt{R F^{2}+C F^{2}}
$$

In the above formula, RFand CF indicate the row and column frequencies, respectively.

$$
\begin{aligned}
& R F=\sqrt{\frac{1}{m n} \sum_{i=1}^{m} \sum_{j=2}^{n}(G(i, j)-G(i, j-1))^{2}} \\
& C F=\sqrt{\frac{1}{m n} \sum_{i=2}^{m} \sum_{j=1}^{n}(G(i, j)-G(i-1, j))^{2}}
\end{aligned}
$$

(6). Make the RBW transform inversion on FULOW and FUHIGH to obtain the low-frequency coefficient of the (k-1)th layer, and fuse this coefficient with the high-frequency of the (k-1)th layer according to steps (4) and (5) until the new first principal components $F C^{1}$ are obtained;

(7). Replace $M C^{1}$ in step (2) by $F C^{1}$, and make the PCA transform inversion on other principal components to get the fused image. 


\section{Experimental Results and Analysis}

Two sets of images with perfect registration were used to estimate the performance of the proposed algorithm.

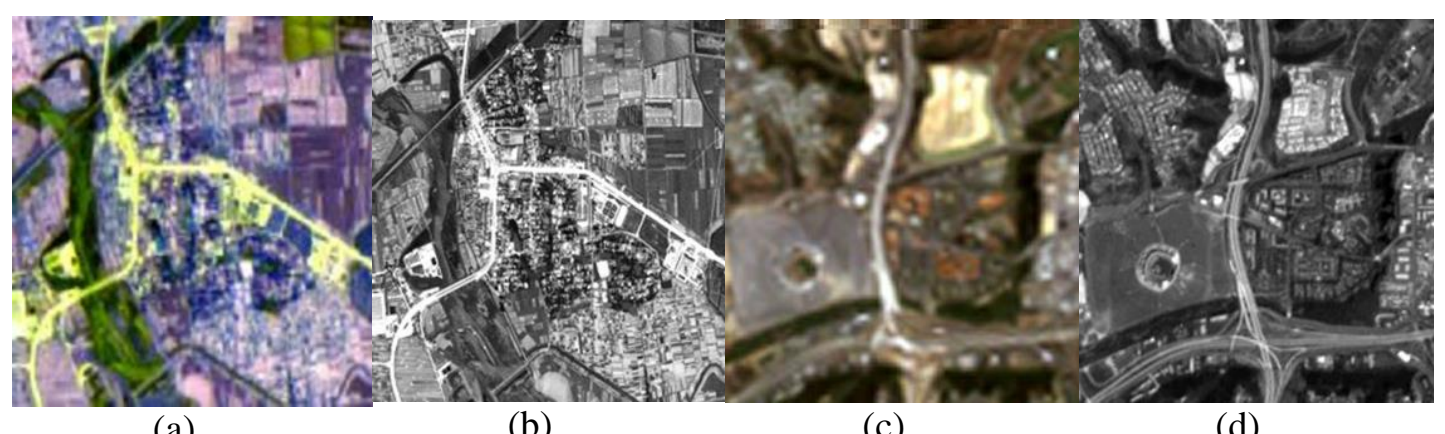

(a)

(b)

(c)

(d)

Figure 5. One Set: (a) Multi-Spectral Image of IKONOS (4 m, 256 $\times 256$ ); (b) Panchromatic Image of IKONOS (1 m, 256 $\times 256$ ); (c) Multi-Spectral Image of Land Sat-TM (30 m, 256 $\times 256$ ); (d) Panchromatic Image of SPOT (10 m, $256 \times 256$ )

\subsection{Parametric Analysis}

Since the information about the main image is contained in the low-frequency portion, the fusion of the low-frequency part is critical. In this section, we discuss the matching threshold $\mathrm{T}$ in the low-frequency fusion algorithm to better preserve the critical features of images. When the reset $\mathrm{T}$ varies from 0.5 to 0.8 , the proposed fusion algorithm was tested with the two sets of images above. The fusion performance was measured by the comprehensive evaluation index (CEI), the degree of distortion (DD), average gradient $(\mathrm{AG})$, and the entropy (EN).

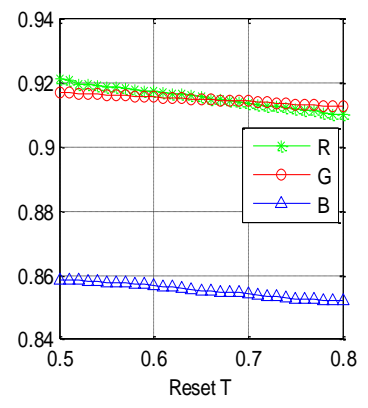

(a) CEI

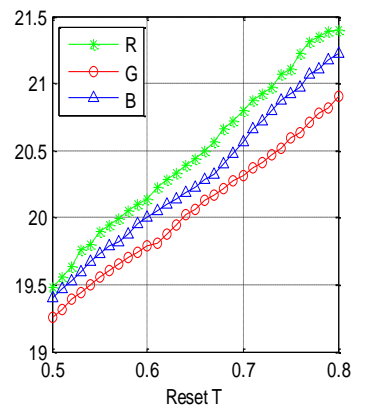

(b) DD

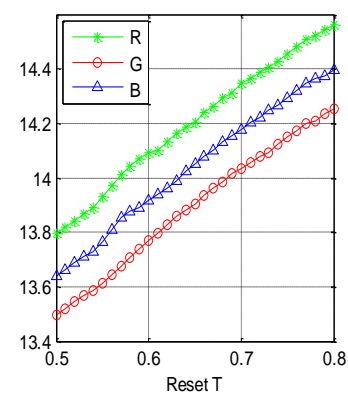

(c) $\mathrm{AG}$

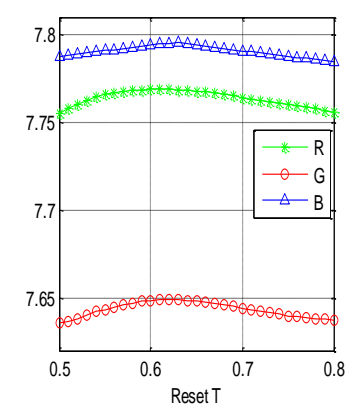

(d) EN

Figure 6. CEI, DD, AG, and EN Change Curves of Image IKONOS 


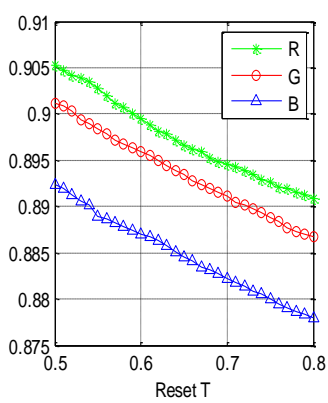

(a) CEI

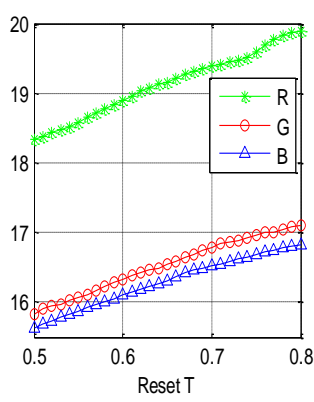

(b) DD

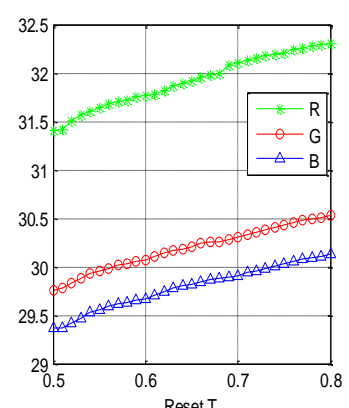

(c) $\mathrm{AG}$

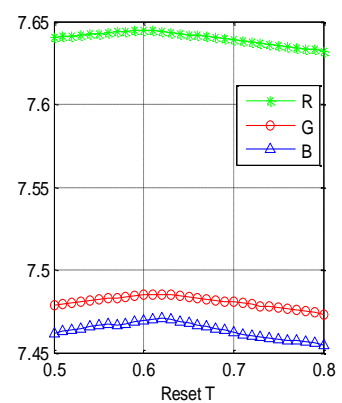

(d) EN

Figure 7. CEI, DD, AG, and EN Change Curves of Images Land sat-TM and SPOT

Based on Figs. 6 and 7, we developed the following conclusions. As the threshold $\mathrm{T}$ is increased gradually, the CEI decreased gradually; entropy tended to decrease after a short period of slight increase, as shown in columns 1 and 3, while the average of the degree of distortion and the clarity increased continuously. To get an optimal balance point between the four evaluation parameters, i.e., CEI, DD, AG, and EN, we set T to 0.65 for IKONOS images, and we set $\mathrm{T}$ to 0.6 for the Land sat-TM and SPOT images.

\subsection{Experimental Results}

In this study, we selected a Land sat-TM multi-spectral image and a SPOT panchromatic image as the experimental images. Images (a) was obtained by IHS transform[4]. The fusion images (b) was obtained by using the PCA transform[3]. Images (c) was obtained by using the wavelet transform (WT)[18] to decomposition. Images (d) was obtained by using RBW transform[19]. The fusion images (e) was based on the fractional B-spline wavelet-based fusion method and PCA (FWT-PCA)[20]. Images (f) was based on the algorithm presented in this paper.

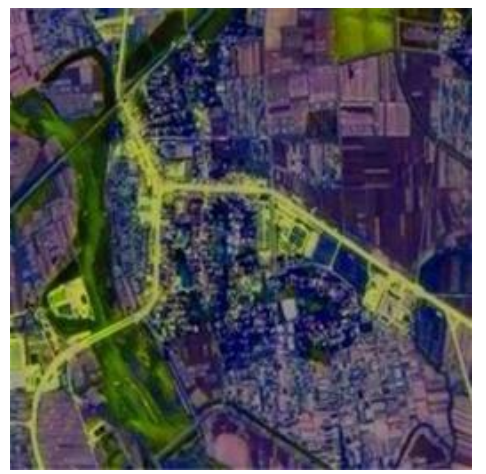

(a)

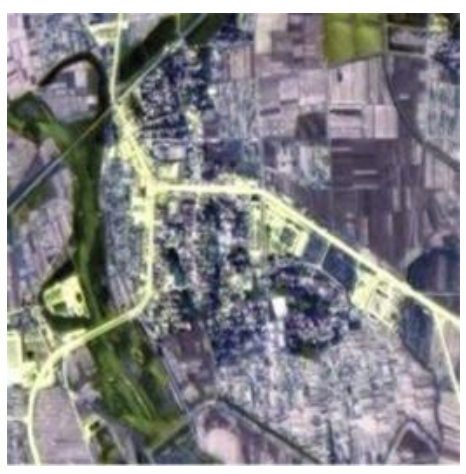

(b)

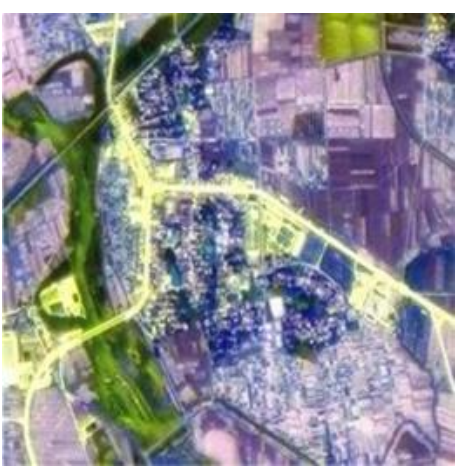

(c) 


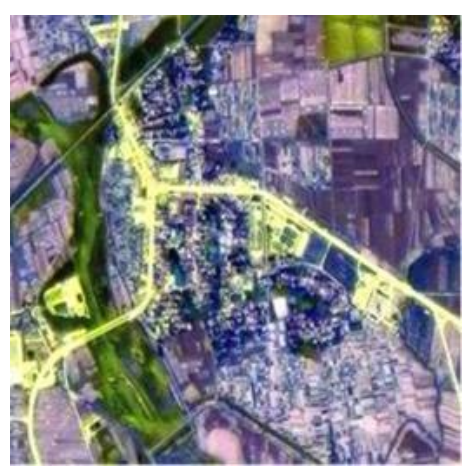

(d)

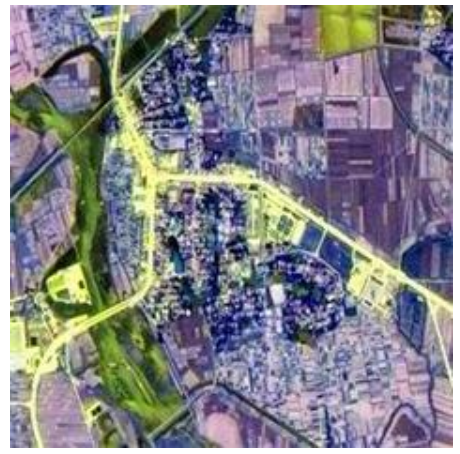

(e)

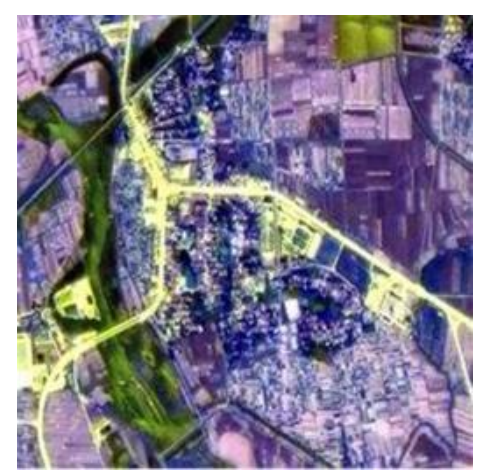

(f)

Figure 8. Fusion image of IKONOS: (a) HIS; (b) PCA; (c) DWT; (d) RBW; (e) FWT-PCA; (f) RBW-PCA

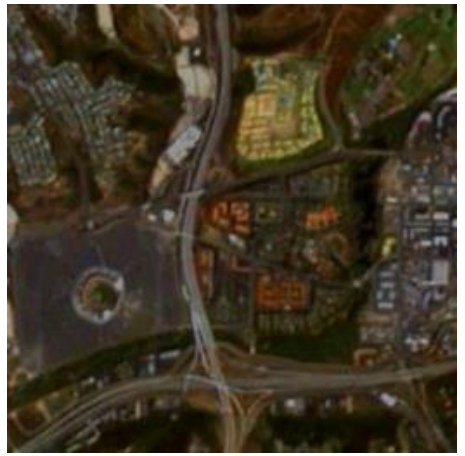

(a)

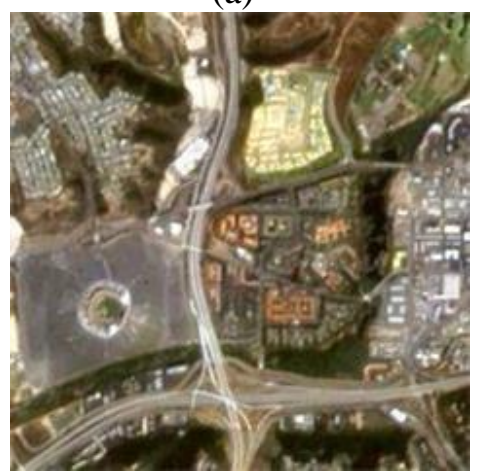

(d)

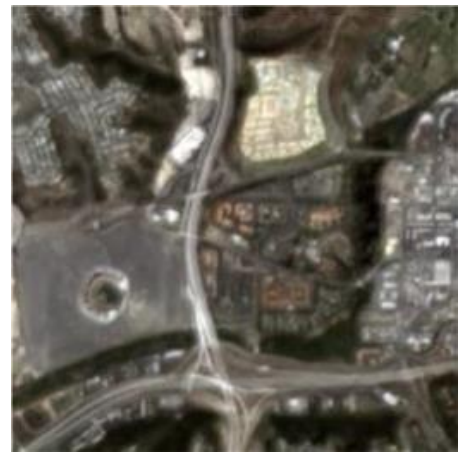

(b)

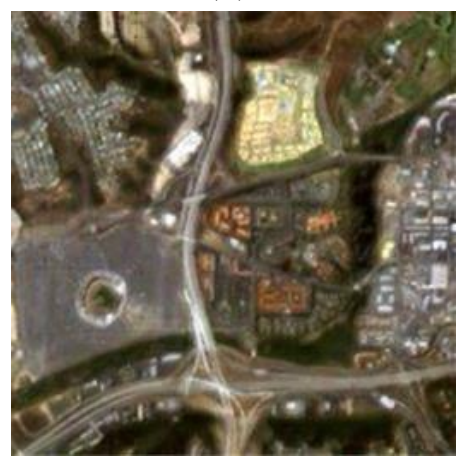

(e)

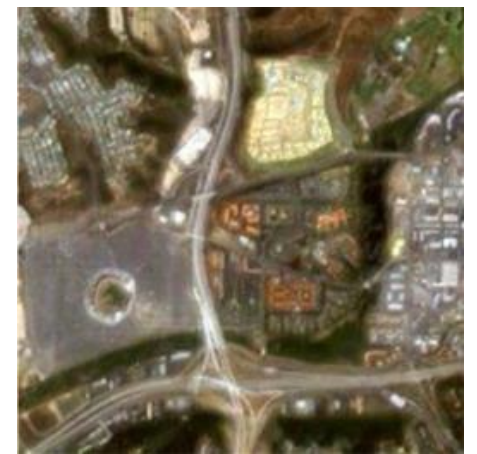

(c)

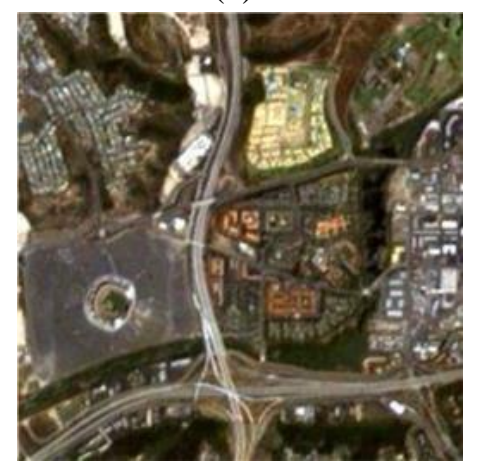

(f)

Figure 9. Fusion image of Landsat-TM and SPOT: (a) HIS; (b) PCA; (c) DWT;(d) RBW; (e) FWT-PCA; (f) RBW-PCA

Visually, the fusion image based on IHS and PCA transform distorted the spectral information and caused some spectral degradation, thereby distorting the color of the fusion image. The fusion image based on the RBW algorithm had the dark-box effect. The image obtained by the FWT-PCA algorithm increased the brightness, but it resulted in a loss of spectral information. The image using the RBW-PCA transform retained a large amount of the spectral information of the MS image and had great clarity. The RBW-PCA achieved far greater improvement in multi-spectral image fusion than the other algorithms. 


\subsection{Objective Quantitative Analysis}

Due to the defects and differences in the human visual system, it is necessary to objectively and quantitatively analyze the effects of the fusion image. To do this, we selected the evaluation of the comprehensive evaluation index, distortions, sharpness, and entropy [21-23].

It was assumed that the sizes of the fused source images $M$ and $P$ were both $m \times n$ ; $M$ indicates the MS image, $P$ indicates the PAN image, and $F$ indicates the fusion image; $\bar{M},(\bar{P})$, and $\sigma_{M}^{2}\left(\sigma_{P}^{2}\right)$ are the grayscale average and the variance of the MS(PAN) image, respectively. The term $\sigma_{M P}$ is the covariance of the fused source images $M$ and $P$.

The impacts of each indicator on the sharpness of the image and on the amount of information are analyzed below:

(1). Standard deviation. The standard deviation reflects the distribution of values of the pixels of the image pixel, and it is proportional to the contrast of the image, i.e., as the standard deviation increases, the contrast of the image increases and the amount of information the image contains increases. If the standard deviation is small, the contrast of the image is lower, which is expressed as:

$$
S D=\sqrt{\frac{1}{m \times n} \sum_{i=1}^{m} \sum_{j=1}^{n}(F(i, j)-\bar{F})^{2}}
$$

(2). The degree of distortion directly reflects the extent to which the spectral information is distorted.

$$
D D=\frac{1}{m \times n} \sum_{i=1}^{m} \sum_{j=1}^{n}|F(i, j)-M(i, j)|
$$

(3). Clarity also is known as the average gradient, which is defined as follows:

$$
\nabla \bar{g}=\frac{1}{(m-1)(n-1)} \sum_{i=1}^{m-1} \sum_{j=1}^{n-1} \sqrt{\left(\left(\frac{\partial F\left(x_{i}, x_{j}\right)}{\partial x_{i}}\right)^{2}+\left(\frac{\partial F\left(x_{i}, x_{j}\right)}{\partial y_{i}}\right)^{2}\right) / 2}
$$

Clarity reflects the contrast of the tiny details and the variations in the texture of the image; the greater the value of clarity becomes, the clearer the image becomes.

(4). Entropy is an important indicator that is used to measure the richness of the information in the image. The amount of information in the fusion image is directly proportional to the entropy.

(5). Comprehensive evaluation index. The fusion image is obtained from two images. It is necessary to consider the relationship between the MS image, the PAN image, and the fusion image $\mathrm{F}$. The comprehensive evaluation index can reflect the degree of relationship between the three images. The definition of the expression is:

$$
\begin{aligned}
& Q(M, P, F)=\lambda Q(M, F)+(1-\lambda) Q(P, F) \\
& Q(M, F)=\frac{\sigma_{M F}}{\sigma_{M} \sigma_{F}} \cdot \frac{2 \bar{M} \bar{F}}{(\bar{M})^{2}+(\bar{F})^{2}} \cdot \frac{2 \sigma_{M} \sigma_{F}}{\sigma_{M}^{2}+\sigma_{F}^{2}} \\
& Q(P, F)=\frac{\sigma_{P F}}{\sigma_{P} \sigma_{F}} \cdot \frac{2 \bar{P} \bar{F}}{(\bar{P})^{2}+(\bar{F})^{2}} \cdot \frac{2 \sigma_{P} \sigma_{F}}{\sigma_{P}^{2}+\sigma_{F}^{2}}
\end{aligned}
$$

The first term shows the linear correlation between $M, P$, and $F$, which varies from -1 to 1 . The second term is the average distortion of the luminance, which varies from 0 to 1. The third term is the average distortion of the contrast, which varies from 0 to 1 . Tables 1 and 2 show the experimental data of the indicators. 
Table 1. Performance Evaluation of IKONOS Image Data Fusion

\begin{tabular}{|c|c|c|c|c|c|c|c|}
\hline \multicolumn{2}{|c|}{$\begin{array}{l}\text { Experimental } \\
\text { Method } \\
\text { Evaluation indicator }\end{array}$} & IHS & PCA & WT & RBW & FWT-PCA & RBW-PCA \\
\hline \multirow{4}{*}{$\begin{array}{l}\text { Comprehensive } \\
\text { evaluation } \\
\text { index }\end{array}$} & $\mathrm{R}$ & 0.8427 & 0.8468 & 0.8865 & 0.8931 & 0.9000 & 0.9157 \\
\hline & G & 0.8402 & 0.8355 & 0.8598 & 0.8516 & 0.8780 & 0.9142 \\
\hline & B & 0.7524 & 0.8047 & 0.7552 & 0.8196 & 0.8410 & 0.8520 \\
\hline & Mean & 0.8118 & 0.8290 & 0.8305 & 0.8547 & 0.8730 & 0.8940 \\
\hline \multirow{4}{*}{$\begin{array}{c}\text { Distorted } \\
\text { degree }\end{array}$} & $\mathrm{R}$ & 26.5072 & 31.0432 & 32.7172 & 28.4558 & 26.5545 & 20.7325 \\
\hline & G & 28.3725 & 27.5282 & 26.8273 & 27.6862 & 22.3392 & 20.4178 \\
\hline & B & 44.1070 & 27.2845 & 25.9371 & 25.5535 & 21.8971 & 20.6373 \\
\hline & Mean & 32.9956 & 28.6188 & 28.8272 & 27.2318 & 23.5969 & 20.5958 \\
\hline \multirow{4}{*}{ Clarity } & $\mathrm{R}$ & 29.9555 & 20.7328 & 26.4191 & 26.6560 & 29.0728 & 35.3812 \\
\hline & G & 29.9455 & 19.1082 & 26.6375 & 27.6021 & 28.9238 & 29.7651 \\
\hline & B & 29.5197 & 18.5465 & 26.6375 & 25.2622 & 27.2876 & 30.0143 \\
\hline & Mean & 29.8069 & 19.4625 & 26.5647 & 26.5068 & 29.7614 & 30.2593 \\
\hline \multirow{4}{*}{ Entropy } & $\mathrm{R}$ & 6.3750 & 6.9374 & 7.4957 & 7.6492 & 7.7393 & 7.7691 \\
\hline & G & 6.3148 & 7.0376 & 7.3953 & 7.5715 & 7.6332 & 7.6490 \\
\hline & B & 6.3015 & 6.9076 & 7.3077 & 7.5635 & 7.7824 & 7.7888 \\
\hline & Mean & 6.3304 & 6.9609 & 7.3996 & 7.5947 & 7.7183 & 7.7356 \\
\hline
\end{tabular}

Table 2. Performance Evaluation of Landsat-TM and SPOT Image Data Fusion

\begin{tabular}{|c|c|c|c|c|c|c|c|}
\hline \multicolumn{2}{|c|}{$\begin{array}{l}\text { Experimental } \\
\text { Method } \\
\text { Evaluation indicator }\end{array}$} & IHS & PCA & WT & RBW & $\begin{array}{c}\text { FWT-P } \\
\text { CA }\end{array}$ & $\begin{array}{l}\text { RBW-P } \\
\text { CA }\end{array}$ \\
\hline \multirow{4}{*}{$\begin{array}{l}\text { Comprehens } \\
\text { ive } \\
\text { evaluation } \\
\text { index }\end{array}$} & $\mathrm{R}$ & 0.7995 & 0.8091 & 0.8727 & 0.8701 & 0.8730 & 0.8946 \\
\hline & G & 0.8066 & 0.7993 & 0.7385 & 0.7823 & 0.8534 & 0.8905 \\
\hline & B & 0.7583 & 0.7976 & 0.8359 & 0.8152 & 0.8251 & 0.8818 \\
\hline & Mean & 0.7881 & 0.8012 & 0.8157 & 0.8225 & 0.8505 & 0.8856 \\
\hline \multirow{4}{*}{$\begin{array}{l}\text { Distorted } \\
\text { degree }\end{array}$} & $\mathrm{R}$ & 27.9351 & 28.3621 & 31.8993 & 24.1178 & 16.4658 & 19.5133 \\
\hline & G & 30.6582 & 25.1076 & 24.9325 & 23.7972 & 15.6724 & 16.7146 \\
\hline & $\mathrm{B}$ & 30.9480 & 25.5632 & 23.3152 & 24.0209 & 23.5573 & 16.5206 \\
\hline & Mean & 29.8271 & 26.3443 & 26.7156 & 23.9786 & 18.5652 & 17.5829 \\
\hline \multirow{4}{*}{ Clarity } & $\mathrm{R}$ & 13.2971 & 9.3337 & 9.7651 & 10.3080 & 13.4495 & 14.3636 \\
\hline & $\mathrm{G}$ & 13.1784 & 9.2711 & 10.0143 & 10.2288 & 13.1554 & 14.0567 \\
\hline & $\mathrm{B}$ & 13.2113 & 9.0785 & 10.2593 & 10.2555 & 13.1554 & 14.2015 \\
\hline & Mean & 13.2271 & 9.2277 & 10.0129 & 10.2641 & 13.2535 & 14.2073 \\
\hline \multirow{4}{*}{ Entropy } & $\mathrm{R}$ & 7.2316 & 7.3225 & 7.4862 & 7.4181 & 7.4784 & 7.6425 \\
\hline & G & 7.0146 & 7.2837 & 7.4075 & 7.3987 & 7.4410 & 7.4818 \\
\hline & B & 7.2541 & 7.2670 & 7.2075 & 7.4137 & 7.4372 & 7. 4727 \\
\hline & Mean & 7.1668 & 7.2910 & 7.3671 & 7.4102 & 7.4522 & 7.5323 \\
\hline
\end{tabular}

The data in Tables 1 and 2 show that the fusion image obtained by the novel algorithm had relatively good spectral information and clarity. And it had the maximum entropy value, which indicated that the image contained a greater quantity of information. The FWT-PCA algorithm improved the texture features of the image by PCA transform and maintained the richness of spectral information by the fractional B-spline wavelet transform, so the brightness and spatial resolution of the fused were enhanced significantly. Also, the degree of distortion was reduced, but there was still some loss of spectral information. The RBW algorithm retains a significant amount of the spectral 
information, but it had a smaller value of clarity. The PCA transform directly replaces the first principal component of the MS image with the first principal component of the PAN image, which increases the clarity and the degree of distortion of the fusion image. The IHS transform directly replaces the luminance component of the MS image with the PAN image, which results in spectral degradation in the fusion image. Therefore, considering the effects on the fusion image, our proposed algorithm provided the best performance.

\section{Conclusions}

A novel, multi-spectral image fusion algorithm based on RBW and PCA is proposed in this paper. The RBW transform decomposes and reconstructs the salient features at different scales, frequency domains, decomposition layers, and frequency bands, which are extracted from the multi-spectral image by the PCA algorithm. We proposed a novel, weighted method based on regional features and local energy to fuse the low-frequency coefficient and an improved method based on neighborhood spatial frequency to fuse the high-frequency coefficients. The fusion image by the proposed algorithm outperformed PCA, IHS, RBW, and FWT-PCA in the following aspects, i.e., it is clearer, contains more information, and maintains greater high-resolution and spectral information. In addition, this algorithm does not have the disadvantages of the traditional wavelet approach, such as depending on the frequency domain, down sampling, and box effects. It also improves the processing speed, takes less memory, and offered the potential for real-time processing.

The RBW performs well in processing sparse information in images, and it will be the topic of future research.

\section{Acknowledgements}

We sincerely appreciate the efforts of those who reviewed this paper, and we express our special thanks to Drs. Zhengzhou Li and Shangbo Zhou, Professors at Chong Qing University, who made effort to improve the paper.

\section{References}

[1] B. Jin, G. Kim and N. I. Cho, "Wavelet-domain satellite image fusion based on a generalized fusion Equation," J. Appl. Remote Sens, vol. 8, no.1, (2014), 080599.

[2] M. M. Huang, J. S. Leng and C. C. Xiang, "A Study on IHS+WT and HSV+WT Methods of Image Fusion,” Int. Symp. Inf. Sci. Eng., ISISE, vol. 8, (2008), pp. 665-668.

[3] M. Gonzalez-Audicana, L. J. Saleta, R. G. Catalan and R. Garcia, "Fusion of multispectral and panchromatic images using improved IHS and PCA mergers based on wavelet decomposition," IEEE Trans. Geosci.Remote Sens., vol. 42, no. 6, (2004), pp. 1291-1299

[4] W. J. Carper, T. M. Lillesand and R. W. Kiefer, "The use of Intensity Hue Saturation transformations for merging SPOT panchromatic and multi-spectral images," Photogrammetric Engineering \& Remote Sens, vol. 56, no. 4, (1990), pp. 459-467.

[5] J. Zhou, D. L. Civco and J. A. Silnde, "A wavelet transform method to merge Land sat TM and SPOT panchromatic data," Int, J, Remote Sens ,vol. 19, no. 4, (1998), pp. 743-757.

[6] J. Wu, J. Liu, J. Tian and B. Yin, "Wavelet-based remote sensing image fusion with PCA and feature product," IEEE Cat. No. 06EX1416C, (2006), pp. 2053-2057.

[7] A. R. Gillespie, A. B. Kahle and R. E. Walker, "Color enhancement of highly correlated images. II. Channel ratio and 'chromaticity' transformation techniques," Remote Sens. Environ., vol. 22, no. 3, (1987), pp. 343-365.

[8] M. Gonzlez-Audcana, X. Otazu, O. Fors and A. Seco, "Comparison between Mallat's and the trous discrete wavelet transform based algorithms for the fusion of multispectral and panchromatic images," Int. J. Remote Sens.,vol. 26, no. 3, (2005), pp. 595-614.

[9] S. Chen, R. Zhang, H. Su and J. Tian, "SAR and Multispectral Image Fusion Using Generalized IHS Transform Based on à Trous Wavelet and EMD Decompositions," IEEE Sensors Journal, vol. 10, no. 3, (2010), pp. $737-745$.

[10] J. Wu, H. Huang, J. Liu and J. Tian, "Remote Sensing Image Data Fusion based on IHS and Local Deviation of Wavelet Transformation," IEEE Cat. No. 04EX911, (2004),pp. 564-568. 
[11] L. Lizong, G. Tiegang, G. Qiaolun and B. Lei, "A Verifiable Copyright-Proving Scheme Based on Lifting Wavelet Transformation," IITSI 2010, (2010), p. 68-72.

[12] S. A. Valizadeh and H. Ghassemian, "Remote sensing image fusion using combining IHS and Curvelet transform," 2012 Sixth International Symposium on Telecommunications, IEEE, Tehran., pp.1184-1189 (2012),

[13] X. Zhao, S. Wang, C. Deng and L. Zhang, "The Directional Adaptive Red-Black Wavelet Transform for Image Coding," ETCS 2009, (2009),pp.148-152.

[14] X. Zhao, S. Wang and C. Deng, "Image Denoising Algorithm Based on the Directional Adaptive Red-Black Wavelet Transform,” 2008 Sec.Int.Sym.on Intelligent Information Technology Application, (2008) , pp. 390-394.

[15] Y. S. Juang, L. T. Ko and J. E. Chen, "Histogram Modification and Wavelet Transform for High Performance Watermarking", Mathematical Problems in Engineering, (2012).

[16] W. Yang, J. Wang and J. Guo, "A Novel Algorithm for Satellite Images Fusion Based on Compressed Sensing and PCA,” Math. Probl. Eng., vol. 33, no. 22, (2013), pp. 7060-7074.

[17] Q. Li, J. Du, F. Song and C. Wang, "Region-based multi-focus image fusion using the local spatial frequency," CCDC 2013, (2013), pp. 3792-3796.

[18] S. Wu, B. Zhao, and M. Bian, "A new fusion method of remote sensing images based on wavelet transform,” ICSP 2012, no. 2, (2012), pp.829-832.

[19] L. Bin,Z. Qing and P. Jiaxiong "Fusion method of multispectral image based on Red-Black wavelet transform", Chinese Journal of Scientific Instrument, vol. 32, no. 2, (2011), pp. 408-414.

[20] W. Yang and Y. Gong, "Multi-spectral and panchromatic images fusion based on PCA and fractional spline wavelet," Int. J. Remote Sens. (UK), vol. 33,no. 22, (2012),pp. 7060-7074.

[21] S. Li, B. Yang and J. Hu, "Performance comparison of different multire solution transforms for image fusion", Information Fusion, vol. 12, no.2, (2011), pp. 74-84.

[22] M. Deshmukh and U. Bhosale, "Image fusion and image quality assessment of fused images," Int J Image Process, vol. 4, (2010), pp.484-508.

[23] S. Klonus and M. Ehlers, "Performance of evaluation methods in image fusion," 2009 12th International Conference on Information Fusion, WA, Seattle,USA, IEEE, (2009), pp. 1409-1416,

\section{Authors}

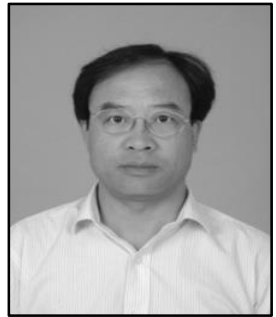

Wenkao Yang, he has received his M.S and PHD degrees in Signal and Information Processing from University of Electronic Science and Technology of China in 1992 and 2003, respectively. After working in the State Administration of Radio Film and Television, he started working at school of Electronic and Information Engineering in Beijing Jiaotong University in 2006.

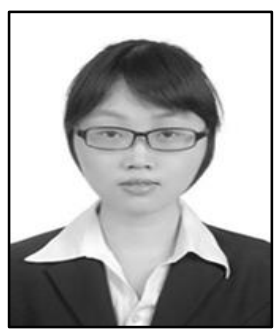

Jing He, she has received his B.S degree from Dalian Polytechnic University in 2013. Now she is studying for the master's degree at the School of Electronic and Information Engineering in Beijing Jiaotong University. And her recently research interests include pattern recognition, image processing.

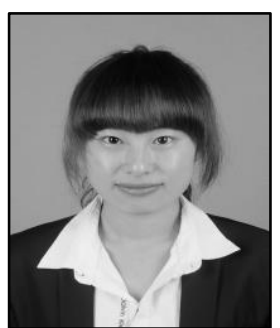

Hongyuan Wang, she has received his B.S degree from Dalian Polytechnic University in 2013. Now she is studying for the master's degree at School of information science and engineering. Her research interests include image processing and communication network. 


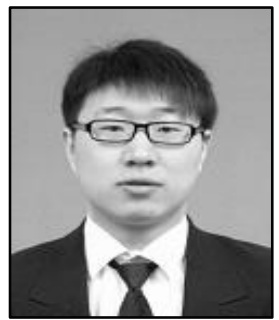

Yue Feng, he has received his B.S. degree at the school of Electronic and Information Engineering in Beijing Jiaotong University in 2013. Now he is studying for his master's degree here. His research interests include pattern recognition, image processing, machine learning. 
International Journal of Signal Processing, Image Processing and Pattern Recognition Vol. 9, No. 5 (2016) 